\title{
Characterization of Polarization Holographic Gratings Obtained on Azopolymer Thin Films by Digital Holographic Microscopy
}

\author{
Veronica Cazac ${ }^{1,2^{*}}$, Constantin Losmanschii ${ }^{1}$, Elena Achimova ${ }^{1}$, Alexey Meshalkin ${ }^{1}$, \\ Vladimir Abashkin ${ }^{1}$, and Vladimir Podlipnov ${ }^{3}$ \\ ${ }^{1}$ Institute of Applied Physics of Moldova, 5 Academiei, Chisinau MD2028, Moldova \\ ${ }^{2}$ Tampere University, Faculty of Information Technology and Communication Sciences, 1 Korkeakoulunkatu, \\ Tampere FI-33720, Finland \\ ${ }^{3}$ Samara National Research University, 34 Moskovskoye shosse, Samara 443086, Russia \\ * e-mail: veronica.cazac@tuni.fi
}

\begin{abstract}
Polarization diffraction gratings are formed by one-step polarization holographic recording in azopolymer thin films. The evolution of the gratings parameters, such as the modulation of diffraction efficiency and relief depth with regard to different exposure dose is analyzed. Phase-shifting digital holographic microscopy is applied for the measurement of the light-induced polarization diffraction gratings. For the accurate hologram acquisition and reconstruction of the complex amplitude transmitted by the gratings, we performed all-optical (without moving components) phase-shifting implemented within in the imaging system of the digital holographic microscope. The experimental measurement results and theoretical predictions were compared and analyzed. (C) 2021 Journal of Biomedical Photonics \& Engineering.
\end{abstract}

Keywords: digital holographic microscopy; polarization holographic gratings; image processing; diffraction efficiency; azopolymer; thin films; phase imaging.

Paper \#3433 received 25 May 2021; revised manuscript received 25 Sep 2021; accepted for publication 25 Sep 2021; published online 30 Sep 2021. doi: 10.18287/JBPE21.07.030306.

\section{Introduction}

Recently, azobenzene-based photochromic materials have attracted great scientific attention due to the light-induced mass migration, which can be used to create polarization holographic optical elements on thin films as a response to patterned illumination. These materials are particularly intriguing because of the reversibility of the photoinduced changes. Periodic structures created on azopolymer surfaces have many different outstanding applications such as visualizing the near-fields of plasmonic structures and the orbital angular momentum of a light beam [1,2]. Interferometry is a flexible approach applied for the creation of complex polarization holographic gratings (PHG) with varying parameters, which would be difficult to create using conventional lithography tools. However, essential understanding and non-destructive observation of light-induced transformations in these materials is crucial and still holds great interest. PHGs represent appropriate test elements for analyzing azopolymer behavior. Digital holographic microscopy (DHM) allows you to visualize not only amplitude information, color information, but also phase information. This is especially useful for obtaining information about living cells, tissues, and various biological materials. [3-5] Digital holographic microscopy (DHM) is a quantitative phase imaging instrument [6], where complex amplitude (amplitude and phase) of light interacting with a sample are measured, by hologram acquisition. Various modifications of such microscopes are known, including a digital holographic white light microscope based on spectral phase shifting [7] DHM can also be used for 3D analysis of the profile of micro-optical components with the highest resolution [8-9].

The hologram is obtained by interference of the wave that passes through the object under study and the reference wave set under an angle in off-axis setup [10], or inline [11] in case of the phase-shifting configuration. In addition, digitalization of the interferograms open the access to the numerical processing of the complex object wavefront for revealing the phase, amplitude and 3D map of the PHG [12]. Optical and physical information about 
a diffraction grating is encoded in the phase component of the digital hologram. This data permits to calculate and deduce different parameters such as the surface relief or refractive index modulation of the recorded optical elements [13-14].

In this paper, we introduce phase-shifting DHM for studying PHG recorded on azopolymer thin films. PHG were created under different recording conditions by polarization holographic recording (PHR). The analysis of the dependence of diffraction efficiency on exposure time and relief depth was carried out. The parameters of PHG obtained via phase-shifting DHM permit to extend the understanding of the light-induced topographical patterns on azomaterial surfaces.

\section{Materials and Methods}

\subsection{Fabrication of azopolymer thin films}

Carbazole-based polymer poly-n-epoxypropylcarbazole (PEPC) and azodye Solvent Yellow 3 (SY3) was applied in this study. SY3 was acquired from Sigma-Aldrich Company. The weight ratio between SY3 and PEPC was 1:3. Azopolymer was obtained by the reflux method for
$8 \mathrm{~h}$, from $0.33 \mathrm{~g}$ PEPC and $0.101 \mathrm{~g} \mathrm{SY} 3 \mathrm{in} 10 \mathrm{ml}$ toluene solution. The obtained solution of carbazole-based azopolymer (PEPC-co-SY3) was filtered. The reaction scheme is illustrated in Fig. 1. Chemical bonding of SY3 to PEPC polymer was performed by a polymerization reaction. Fabrication of the azopolymer based thin films was done via spin-coating. The main advantages of this method are uniformity and high spinning speed resulting in thin layers' deposition on a micrometer scale. The thickness of the synthesized azopolymer was $1.035 \pm 0.027 \mu \mathrm{m}$. The measurement was carried out digitally on a modified MII-4 interferometer [15].

\subsection{Recording of PHGs on azopolymer thin films}

Photo-induced surface relief modulation in azopolymer thin films is closely linked to the sensibility of azobenzene compounds to the polarization of light. It was proved that the different light induced phenomena in azopolymer materials appear at a macroscopic level by a series of transcis-trans isomerizations cycles when their chromophores reorient perpendicularly to the electric vector of linearly polarized light [16].
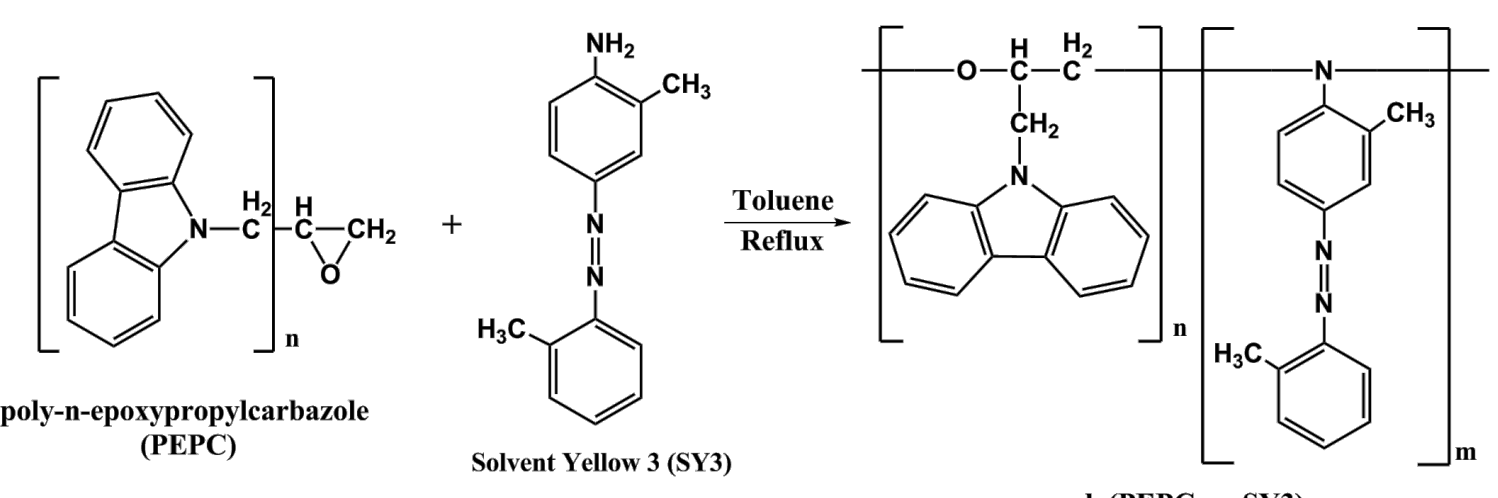

Fig. 1 Synthetic route of PEPC:SY3 azopolymer synthesis.

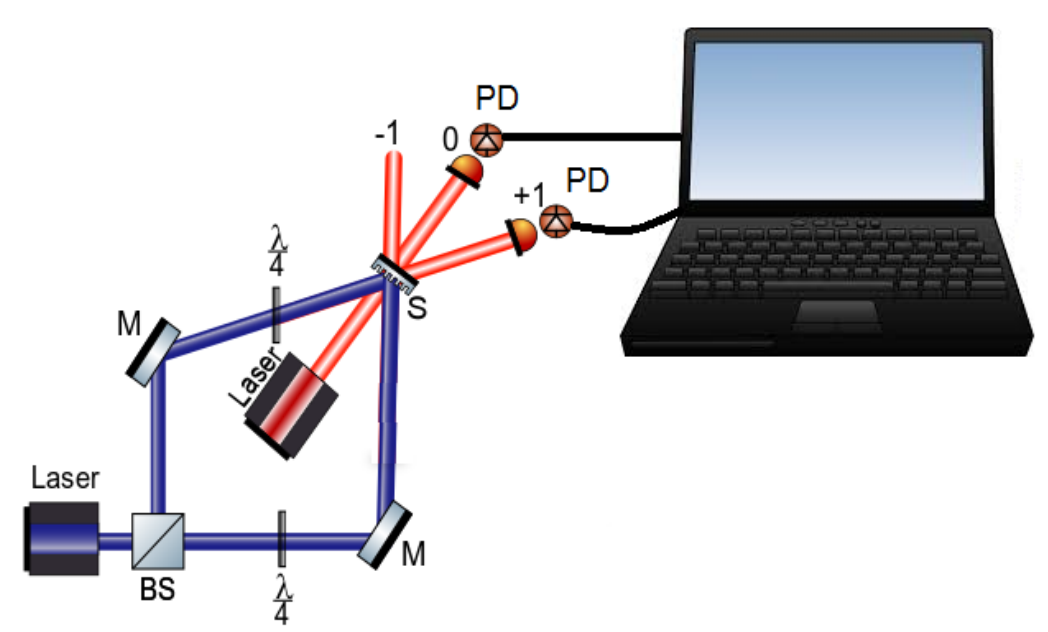

Fig. 2 Polarization holographic recording setup: laser-single mode DPSS $473 \mathrm{~nm}(100 \mathrm{~mW})$, BS-polarized beam splitter, Mmirrors, $\lambda / 4$-quarter wave plate, Laser $650 \mathrm{~nm}$, S-azopolymer thin film, PD-photodiodes for DE registration. 


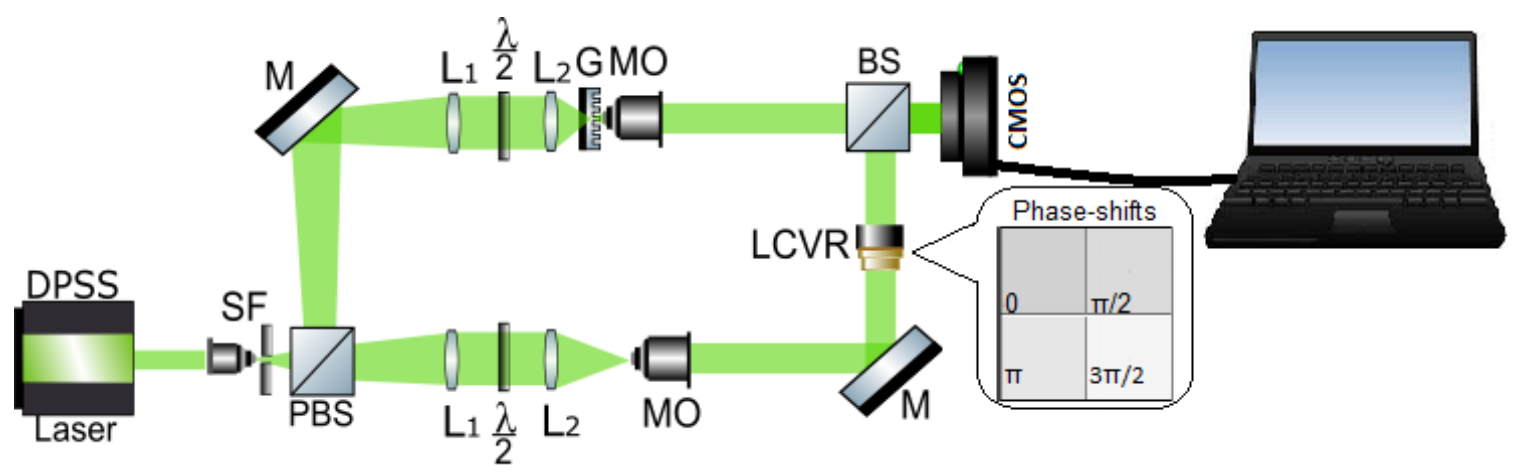

Fig. 3 The setup of the phase-shifting DHM: Laser-single mode DPSS $473 \mathrm{~nm}(100 \mathrm{~mW}), \mathrm{SF}$ - spatial filter, PBS - polarized beam splitter, M-mirrors, $\lambda / 2$-halve wave plate, lenses L1, L2, G-grating, MO - microobjectives, LCVR - liquid-crystal variable retarder, BS beam splitter, CMOS - digital CMOS camera.

Diffraction gratings for different exposures were made by the polarized holographic recording method. The optical setup is shown in Fig. 2. The recording laser is single mode DPSS laser (CNI Lasers MSL-FN-473), $\lambda=473 \mathrm{~nm}, 100 \mathrm{~mW}$. The polarization of the incident beams was LCP : RCP was obtained by $\lambda / 4$ wave retarders. To evaluate the performance of the obtained PHGs the diffraction efficiency (DE) evolution has been measured by two photodiodes and a red laser HLM1230, $\lambda=650 \mathrm{~nm}, 5 \mathrm{~mW}$ which does not modulate the azopolymer film. DE was determined by the following relation:

$$
\eta=\frac{I_{1}}{2 I_{1}+I_{0}} \times 100 \%,
$$

where, $I_{0}$ is the light intensity from zero order, and $I_{1}$ is the light intensity in first diffraction order, respectively.

Both interfering beams pass through quarter-wave plates for obtaining a polarized DOE. The angle between interfering beams is $\theta \approx 40^{\circ}$ and the period of the grating on the $\mathrm{X}$-axis $\Lambda=\lambda / 2 \sin \frac{\theta}{2}$ is about $7 \mu \mathrm{m}$.

\subsection{DHM investigations}

The setup of the phase-shifting DHM is illustrated in Fig. 3. In the experiment, the single-mode laser beam (CNI Lasers MSL-FN-473) is divided by a polarized beamsplitter into the reference wave- $R_{w}$ and the object wave that passes through the PHG- $\mathrm{O}_{\mathrm{w}}$. Laser beam diameters are controlled by two lenses $\mathrm{L}_{1}, \mathrm{~L}_{2}$. The collimated beams pass through a half-wave plates to adapt the polarization state of the reference wave to the state of the object waves. Identical microscopic optical systems are set in both paths. The CMOS camera acquires digital holograms which represents the in-line interference pattern of beams. Four phase-shifts equal to $\varphi_{1}=0, \varphi_{2}=\pi / 2, \varphi_{3}=\pi, \varphi_{4}=3 \pi / 2$ are produced by the LCVR (Meadowlark LVR-100 VIS), detailed calibration and explanation of the working procedures are described in Ref. [16]. The phase step is equal to $\pi / 2$.
For reconstructing the map surface relief modulation of the PHGs four object holograms are captured within the grating recorded in the azopolymer film. Another four reference holograms are recorded on the area of the film that was not exposed to electromagnetic radiation. This subtraction ensures the reduction of the background noise. Finally, the phase map of the PHG is calculated by the following formula:

$$
\Delta \Phi(x, y)=\Delta \Phi_{0}(x, y)-\Delta \Phi_{R}(x, y)
$$

where $\Delta \Phi_{0}(x, y), \quad \Delta \Phi_{R}(x, y) \quad$ represent the reconstructed phase maps from the object and reference holograms, respectively.

\section{Results and Discussions}

The quality of the and surface light-induced deformations in PHGs is important for estimating the azopolymer thin films behavior under the influence of light interference. The surface relief depth $(\Delta h)$ of the PHG is calculated numerically in MATLAB software from the unwrapped phase by

$$
\Delta h(x, y)=\frac{\Delta \Phi(x, y)}{2 \pi(n-1)} \lambda,
$$

where $\lambda=532 \mathrm{~nm}$ is the wavelength and $\mathrm{n}=1.6$ is the refractive index of the azopolymer films.

Since refractive index modulation is small in azopolymer thin films usually, it suffices to consider only the surface relief modulation produced by modifications in the volume of the azopolymer [15]. Thus, in our calculations it is assumed that the refractive index coefficient is not changed during recording.

Theoretical calculations and experimental DHM measurement results of the PHGs obtained using differed exposure doses are illustrated in Figs. 4 and 5. The obtained gratings have a sinusoidal form. The maximal height modulation according to theoretical calculations is reached when the azopolymer is exposed for $30 \mathrm{~min}$, the 
value of height for this PHR is equal to $780 \mathrm{~nm}$ as shown by the red curve in Fig. 4. However, according to the DHM measurements the maximal modulation constitutes $650 \mathrm{~nm}$ corresponding to $12 \mathrm{~min}$ recording time.

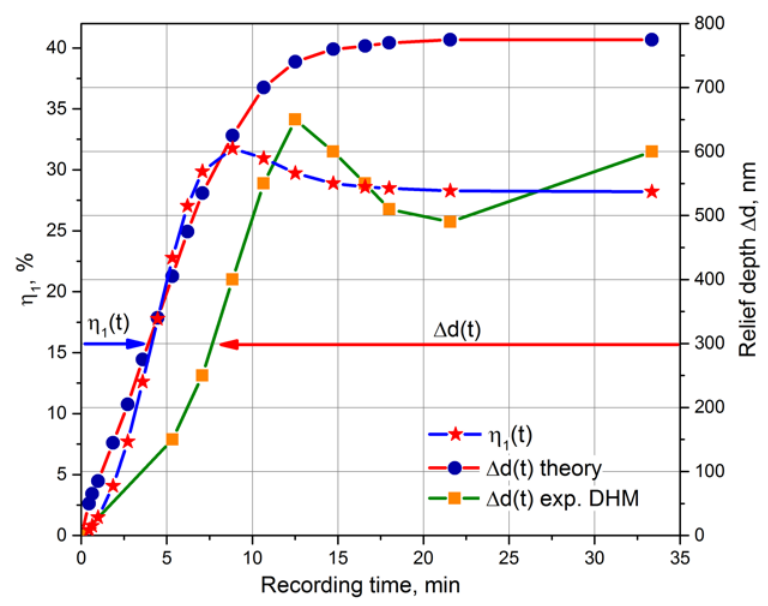

Fig. 4 Kinetics of the first-order DE of the recorded PHGs (blue), relief depth calculated theoretically (red), relief depth measured experimentally obtained by phase-shifting DHM (green).

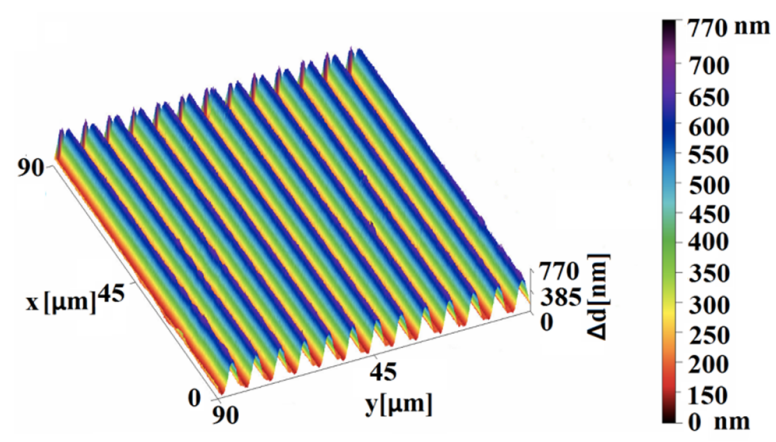

(a)

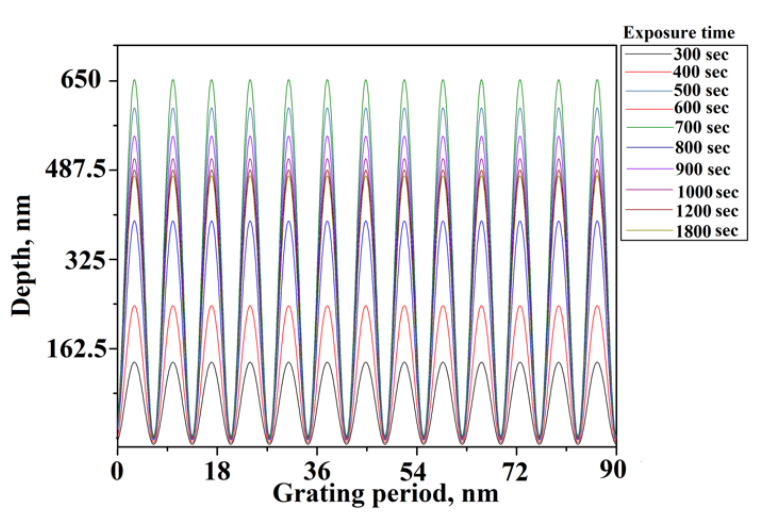

(b)

Fig. 5 Theoretical calculations and experimental DHM measurement results of the PHGs: (a) 3D topography map of the PHG with the maximal obtained height, (b) Crosssections taken across $\mathrm{X}$-axis of the PHGs.
Discrepancies are also observed in the evolution of the relief depth. DHM measurements indicate that the height of the relief decreases after $12 \mathrm{~min}$ and starts to increase again after $27 \mathrm{~min}$ of exposure, while theoretical calculations show a constant growth, until a certain point of saturation. Remarkably, the evolution of the relief modulation defined by DHM corresponds to the kinetics of the first-order DE measured by the photo-diodes during the recording process and represented by the blue curve in Fig. 4. The kinetics reveals that maximal DE is achieved within 8 min of exposure. Fig. 5(a) illustrates the 3D topography map obtained from DHM measurements of the PHG with the maximal relief height equal to $650 \mathrm{~nm}$. According to the cross-sections of the PHRs, illustrated in Fig. 5(b) the grating period is approximately $7 \mu \mathrm{m}$, which corresponds to the experimental recording conditions.

\section{Conclusion}

We have presented the procedures for micropatterning under different exposure doses of PHGs on carbazolebased azopolymers. The interferometric approach for design and characterization of the created patterns has proven to be a versatile technique. In order to realize the full potential of PHG and understand their formation dynamics and response to different exposure doses we used theoretical calculations and experimental measurements using phase-shifting DHM. The comparison between obtained experimental and theoretical parameters of the recorded PHG show particular differences in the relief depth values.

We have two assumptions on the background of this results. First, we assume that the variations in the results may be due to the refractive index changes also arising in azopolymer thin films during exposure. Second, the nonuniform modulations of the relief depth of the PHGs caused by the Gaussian beam distribution creates a difficulty to investigate the gratings in the area with maximal depth modulation [17]. Therefore, further investigations in this field would increase the knowledge on the mechanisms of photoinduced phenomena in azopolymer thin films into the phase modulation of the azopolymer thin films would further facilitate complete understanding of the behavior as well as the photonic applications of these materials, including as a sensitive medium for polarization. These materials can be useful in biomedical research. Along with the well-known methods of visualization of color, shape, and spectral properties using hyperspectral imaging microscopy [18] or Raman spectroscopy of light reflected from a micropreparation [19], the method of digital holographic microscopy based on phase reconstruction can be extremely useful in morphological studies of microobjects.

\section{Disclosures}

All authors declare that there is no conflict of interests in this paper. 


\section{Acknowledgments}

This work was partially supported by The World Federation of Scientists National Scholarship Programme,
Content NARD of Republic of Moldova BIOHOLO project 20.70086.16/COV (01.07.2020-30.06.2021) and partially supported by "ERA.Net RUS plus" program and funded by RFBR, project number 20-52-76021.

\section{References}

1. H. Rekola, H. A. Berdin, C. Fedele, M. Virkki, and A. Priimagi, "Digital holographic microscopy for real-time observation of surface-relief grating formation on azobenzene-containing films," Scientific Reports 10(1), 19642 (2020).

2. A. Meshalkin, C. Losmanschii, A. Prisacar, E. Achimova, V. Abashkin, S. Pogrebnoi, and F. Macaev, “Carbazolebased azopolymers as media for polarization holographic recording," Advanced Physical Research 1(2), 86-98 (2019).

3. A. Anand, V. K. Chhaniwal, N. R. Patel, and B. Javidi, “Automatic Identification of Malaria-Infected RBC With Digital Holographic Microscopy Using Correlation Algorithms,” IEEE Photonics Journal 4(5), 1456-1464 (2012).

4. A. S. Singh, A. Anand, R. A. Leitgeb, and B. Javidi, "Lateral shearing digital holographic imaging of small biological specimens," Optics Express 20(21), 23617-23622 (2012).

5. U. Schnars, W. Jueptner, Digital Holography: Digital hologram recording, numerical reconstruction and related techniques, Springer, Berlin, Heidelberg (2005). ISBN: 978-3-540-26911-3.

6. V. Cazac, A. Meshalkin, E. Achimova, V. Abashkin, V. Katkovnik, I. Shevkunov, D. Claus, and G. Pedrini, "Surface relief and refractive index gratings patterned in chalcogenide glasses and studied by off-axis digital holography," Applied Optics 57(3), 507-513 (2018).

7. D. Kim, J. W. You, and S. Kim, "White light on-axis digital holographic microscopy based on spectral phase shifting," Optics Express 14(1), 229-234 (2006).

8. W. Yunxin, W. Dayong, Z. Jie, L. Yan, and W. Yuhong, "3D profile measurement for micro-optical component by using digital holographic microscopy,” Acta Optica Sinica 31(40), 117-122 (2011).

9. Q. Lin, D. Wang, Y. Wang, S. Guo, S. Panezai, L. Ouyang, and J. Zhao, "Super-resolution quantitative phase-contrast imaging by microsphere-based digital holographic microscopy," Optical Engineering 6(3), 034116 (2017).

10. Z. Monemhaghdoust, F. Montfort, Y. Emery, C. Depeursinge, and C. Moser, "Off-axis digital holographic camera for quantitative phase microscopy," Biomedical Optics Express 5(6), 1721 (2014).

11. M. Trusiak, J. A. Picazo-Bueno, P. Zdankowski, and V. Micó, "DarkFocus: numerical autofocusing in digital in-line holographic microscopy using variance of computational dark-field gradient," Optics and Lasers in Engineering 134, 106195 (2020).

12. V. Cazac, "Improved 3D imaging of phase shifting digital holographic microscope by compensation for wavefront distortion," Journal of Physics: Conference Series 1745(1), 012020 (2021).

13. A. Anand, I. Moon, and B. Javidi, "Automated Disease Identification With 3-D Optical Imaging: A Medical Diagnostic Tool," Proceedings of the IEEE 105(5), 924-946 (2017).

14. S. Rawat, S. Komatsu, A. Markman, A. Anand, and B. Javidi, "Compact and field-portable 3D printed shearing digital holographic microscope for automated cell identification," Applied Optics 56(9), D127-D133 (2017).

15. A. Meshalkin, A. Andries, E. Achimova, L. Bets, I. Andries, and S. Drahnea, "Interferometric Method Application for Sub-micrometers Thickness Measurements of Spin-coated PEPC and PETPC Polymer Films," in International Conference on Nanotechnologies and Biomedical Engineering, Chişinău, Republic of Moldova, 194-197 (2011).

16. A. Priimagi, A. Shevchenko, "Azopolymer-based micro- and nanopatterning for photonic applications," Journal of Polymer Science Part B: Polymer Physics 52(3), 163-182 (2014).

17. V. Cazac, E. Achimova, V. Abashkin, A. Prisacar, C. Loshmanschii, A. Meshalkin, and K. Egiazarian, "Polarization holographic recording of vortex diffractive optical elements on azopolymer thin films and 3D analysis via phaseshifting digital holographic microscopy," Optics Express 29(3), 9217-9230 (2021).

18. O. V. Polschikova, A. S. Machikhin, A. G. Ramazanova, I. A. Bratchenko, V. E. Pozhar, I. V. Danilycheva, and M. V. Danilychev, "An acousto-optic hyperspectral unit for histological study of microscopic objects," Optics and Spectroscopy 125(6), 1074-1080 (2018).

19. L. A Bratchenko, I. A. Bratchenko, A. A. Lykina, M. V. Komarova, D. N. Artemyev, O. O. Myakinin, A. A. Moryatov, I. L. Davydkin, S. V. Kozlov, and V. P. Zakharov, "Comparative study of multivariative analysis methods of blood Raman spectra classification,” Journal of Raman Spectroscopy 51(2), 279-292 (2020). 